\title{
Abordagens Exata e Heurística para o Mapeamento de Redes Virtuais
}

\author{
Autor: Samuel M. A. Araújo ${ }^{1}$ \\ Orientador: Geraldo R. Mateus ${ }^{1}$ \\ Coorientadora: Fernanda S. H. de Souza ${ }^{2}$
}

${ }^{1}$ Departamento de Ciência da Computação

Universidade Federal de Minas Gerais (UFMG) - Belo Horizonte, MG - Brasil

${ }^{2}$ Departamento de Ciência da Computação

Universidade Federal de São João del-Rei (UFSJ) - São João del-Rei, MG - Brasil

smaa@dcc.ufmg.br, fsumika@ufsj.edu.br, mateusldcc.ufmg.br

\begin{abstract}
Resumo. A virtualização de redes é uma técnica que permite a emulação de diferentes redes virtuais (VNs) simultaneamente sobre o mesmo substrato físico de rede (SN). Tal técnica induz benefícios inerentes à liberdade dos protocolos rígidos de rede, além de facilitar o desenvolvimento de novas tecnologias web. Definir o melhor conjunto de equipamentos do SN para hospedar as $V N s$ é um problema pertencente à classe NP-difícil, denominado Mapeamento de Redes Virtuais (VNE). Diversamente à literatura corrente, este trabalho se singularizou por ter apresentado diferentes abordagens exata e heurística para a resolução do VNE, atuando em ambientes compostos por um ou mais domínios de rede. Adicionalmente, as abordagens desenvolvidas conseguem atender às diferentes características das demandas de VNs, transitando entre os modelos de processamento online, periódico e offline. Neste trabalho ainda foi proposta a aplicação da abordagem heurística em um ambiente multidomínio e online, através do controle de um orquestrador de rede. Um estudo comparativo de três modelos de orquestração distintos foi realizado, sendo com: (i) conhecimento total, (ii) conhecimento parcial e (iii) sem conhecimento da infraestrutura de rede física interna aos domínios. Ao final foram comparados e discutidos os comportamentos das abordagens propostas, usando quatro diferentes objetivos: (i) balanceamento de carga, (ii) consumo de energia elétrica, (iii) lucro dos provedores de serviço e (iv) troca de mensagens entre diferentes domínios.
\end{abstract}

\section{Introdução}

A internet evoluiu muito desde sua concepção, não só em relação à infraestrutura física, mas também quanto a sua utilidade. Dessa forma, a internet hoje é vista como um pilar da sociedade moderna, capaz de centralizar e prover facilmente ao usuário informações sobre diferentes questões como cultura, sociedade, política etc. O notado crescimento da internet encontra dificuldades inerentes ao fato da internet ser originalmente desenvolvida sobre protocolos rígidos e limitados. Um dos problemas observados, é que existe um grande número de diferentes aplicações operando sobre a internet, na qual requerimentos específicos como segurança, escalabilidade, flexibilidade, mobilidade e isolamento, podem não ter sido previstos na criação dos protocolos originais. Assim, surgem desafios como a demanda por links com segurança e disponibilidade, e que atendam aos diferentes 
requisitos demandados pelas aplicações. Tal problema, misturado à rigidez dos protocolos que não permitem alterações em seu núcleo, é chamado de "ossificação da internet" [Zhu and Ammar, 2006].

Uma alternativa para a mitigação dos problemas acarretados pela ossificação da internet é a aplicação de mecanismos de virtualização de redes. Tais mecanismos permitem a implementação de novas funcionalidades às aplicações web, ao se criar uma visão lógica do hardware, de forma que múltiplas VNs com características particulares possam compartilhar o mesmo SN. Nesse contexto emerge o problema conhecido como Mapeamento de Redes Virtuais (VNE), que consiste em determinar o mapeamento das VNs, compostas por roteadores e enlaces virtuais, sobre o SN, composto por roteadores e enlaces físicos [Fischer et al., 2013]. O problema de se mapear uma ou mais VNs, com demandas de recursos não nulas sobre um SN com capacidades de ofertas limitadas, é tido como pertencente à classe dos problemas NP-difíceis [Zhu and Ammar, 2006].

\subsection{Virtualização de Redes}

O desafio encontrado no VNE é alocar de forma eficiente os componentes ofertados pelo SN, de maneira a atender às demandas de VNs dos clientes. Desse modo, o VNE recebe como entrada um conjunto de VNs, sendo cada VN composta por nós e enlaces virtuais, que devem ser aninhados sobre nós e enlaces físicos, que compõem o SN. Como saída o algoritmo deve prover a tomada de decisão quanto a aceitação ou rejeição da(s) $\mathrm{VN}(\mathrm{s})$, sendo que em caso de aceitação o mapeamento deve ser realizado de acordo com a disponibilidade dos recursos residuais do SN no momento do seu processamento.

Diversas variações são admitidas para o problema VNE. Na versão tratada neste trabalho, foram consideradas capacidades de processamento e largura de banda de transmissão, nos nós e enlaces físicos respectivamente. De forma similar, as VNs possuem demandas de processamento associadas a cada nó virtual e de largura de banda de transmissão nos enlaces. Cada nó virtual de uma VN deve ser mapeado em um único nó do SN que deve estar dentro de uma localização válida, definida por um raio de mapeamento do nó virtual em questão, sendo que um mesmo nó físico não deve hospedar mais de um nó virtual de uma mesma VN. Por outro lado, um enlace virtual pode ser mapeado em mais de um enlace físico, isto é, pode ser mapeado em um caminho físico.

O modelo de negócio tradicional do VNE envolve algumas entidades distintas, entre elas: os Provedores de Serviço de Internet (ISPs), responsáveis por gerir os componentes dos domínios, além de instanciar e gerir as VNs; e os usuários finais, que consomem os serviços prestados pelos ISPs [Chowdhury et al., 2009, Fischer et al., 2013]. Neste trabalho, um domínio de rede foi definido como um conjunto de equipamentos de infraestrutura de redes gerenciados por um ISP, podendo ser composto por swiches, hubs, roteadores, fibra ótica, cabo coaxial etc

Um relevante desafio acerca do VNE, abordado e tratado nesta dissertação, consiste na composição do $\mathrm{SN}$, que pode ser formado por um único domínio (SD) ou pela união de vários domínios (MD). Diversos trabalhos desenvolvidos abordam o mapeamento $\mathrm{SD}$, no qual os recursos de rede são regidos por políticas intrínsecas a um único ISP. Em uma abordagem MD, podem estar envolvidos vários ISPs, onde cada ISP administra seus recursos de rede de acordo com políticas internas, a fim de maximizar seus interesses de forma independente [Houidi et al., 2011, Samuel et al., 2013]. Uma das 
premissas inerentes a essa situação é estipular políticas de comunicação entre domínios que respeitem as políticas locais de cada ISP. Um dos desafios desta dissertação foi gerar bons mapeamentos que atuem tanto em ambientes SD quanto MD. A Figura 1 mostra um conjunto de VNs a serem mapeadas num cenário MD.

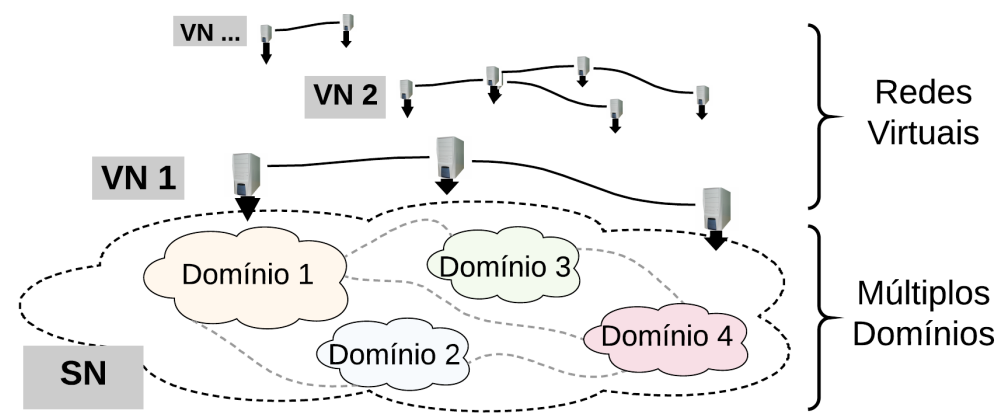

Figura 1. Representação do mapeamento de redes virtuais MD

Agregado ao problema de se propor uma abordagem eficiente para resolver o VNE, tanto SD quanto MD, outros desafios atuais foram considerados e avaliados neste trabalho: maximização do balanceamento de carga, onde o objetivo é melhorar a utilização e diminuir a fragmentação nos componentes do SN; minimização do gasto com energia elétrica, tratamento que segue uma linha de consciência ambiental, de grande importância em função do alto consumo de energia dos ISPs; minimização da troca de mensagens entre domínios diferentes, o que pode melhorar o overhead de comunicação ${ }^{1}$; e maximização do lucro dos ISPs envolvidos.

Em menção ao modelo de processamento das VNs (um dos estudos diferenciais proposto neste trabalho), aplica-se o termo online, offline e periódico em referência à chegada das VNs ao provedor e seus respectivos modos de processamento [Fischer et al., 2013]. O contexto online é caracterizado pela chegada das VNs de maneira aleatória e desconhecida, onde não se sabe de nenhum aspecto sobre a topologia, tempo de vida e requisitos demandados. Neste caso, a VN não sofre atraso de mapeamento, visto que a mesma é processada de imediato. No exemplo da Figura 2, as VNs são processadas na ordem $V=\{\{V N 1\},\{V N 2\},\{V N 3\},\{V N 4\},\{V N 5\}\}$, através de 5 execuções do algoritmo de mapeamento, uma separada para cada $\mathrm{VN}$.

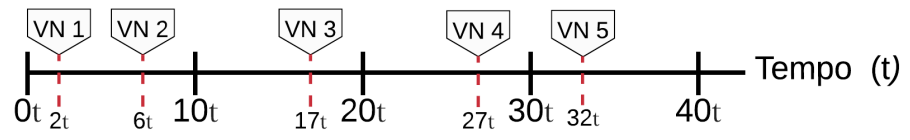

Figura 2. Exemplo do tempo de chegada das requisições

$\mathrm{O}$ contexto offline efetua o mapeamento de um único lote de VNs simultaneamente; neste caso o algoritmo beneficia-se do fato de conhecer integralmente as topologias, tempos de vida e requisitos. No exemplo da Figura 2, o lote de requisições tem $5 \mathrm{VNs}$, composto por $V=\{V N 1, V N 2, V N 3, V N 4, V N 5\}$.

O modelo periódico pode ser concebido como uma abordagem intermediária entre os tratamentos online e offline, no qual uma janela de tempo é adotada para o

\footnotetext{
${ }^{1}$ Refere-se a um consumo extra de banda em um enlace e potenciais atrasos gerados pela troca de mensagens entre domínios diferentes para se prover um mapeamento.
} 
processamento das VNs. De acordo com a duração da janela de tempo, são definidos os lotes que podem conter um número variado de VNs. No exemplo da Figura 2, considerando a duração da janela tempo como 10t, o algoritmo de mapeamento é executado por 4 vezes, sendo $V=\{\{V N 1, V N 2\},\{V N 3\},\{V N 4\},\{V N 5\}\}$. Este modelo gera atrasos, pois os lotes de VNs devem esperar a janela de tempo encerrar para serem processados. Ao final, no exemplo da Figura 2 o atraso total é de $26 t$.

Dado o desafio de atuar em um ambiente MD, este trabalho ainda apresentou um orquestrador de rede que assume um papel de intermediação na contratação de recursos dos ISPs para o mapeamento das VNs requisitadas. Neste trabalho foram propostos três modelos de orquestração distintos: (i) com conhecimento total, (ii) parcial e (iii) sem conhecimento da infraestrutura de rede física interna aos domínios, sendo os três casos analisados junto a uma abordagem heurística de rápida execução.

\section{Objetivos}

A dissertação teve como objetivo geral propor diferentes abordagens para o VNE em um único domínio (VNESD) e em múltiplos domínios (VNEMD), e que concomitantemente atendam de maneira online, periódica e offline as demandas de VNs. De modo específico, na dissertação, buscou-se desenvolver um modelo matemático em Programação Linear Inteira (PLI) para resolução exata do VNE; propor uma abordagem rápida baseada em metaheurísticas; definir um conjunto de políticas de mapeamento para maximizar o lucro e o balanceamento de carga, e minimizar o gasto de energia elétrica e a troca de mensagens; analisar o desempenho da abordagem heurística, comparando com os resultados gerados pelo modelo em PLI; e analisar o desempenho da abordagem heurística junto a algumas variações propostas de um orquestrador de rede.

\section{Soluções Propostas e Contribuições}

$\mathrm{Na}$ dissertação foram desenvolvidas e apresentadas duas abordagens para resolução do problema VNE, sendo tais abordagens aptas para atuar tanto no contexto online, quanto periódico ou offline. Tais abordagens foram: (i) Exata, baseada em PLI e utilizada para encontrar uma solução ótima, porém com tempo impraticável para instâncias de grande porte; (ii) Heurística, chamada de GRASPF-RVNS, baseada na metaheurística Greedy Randomized Adaptive Search Procedure (GRASP) com filtro para construir as soluções iniciais, e combinada com uma busca local a partir de uma variação da metaheurística Variable Neighborhood Search (VNS). Tal abordagem se carateriza por ser computacionalmente eficiente, buscando encontrar uma solução de boa qualidade em um tempo viável.

A fim de atuar nos diferentes contextos de processamento das VNs, foi implementado um simulador de eventos para gerenciar as chegadas das requisições e estabelecer a atuação do algoritmo de mapeamento. Da mesma forma, as requisições são encerradas após terem seu tempo de vida expirado, devolvendo ao SN os recursos alocados anteriormente. Todo controle é realizado pelo simulador na realização dos experimentos computacionais.

Em nossa abordagem, o orquestrador de rede proposto assume um papel de intermediação na contratação dos serviços dos ISPs pelos clientes finais. São apresentados três modelos de orquestração distintos: (i) com conhecimento total, (ii) parcial e (iii) sem 
conhecimento da infraestrutura de rede física interna aos domínios. Em nosso modelo, a cada requisição de VN feita por um usuário final, o orquestrador contrata os serviços dos domínios com garantia de conexão por um determinado tempo estabelecido.

Com base nas abordagens mencionadas, foram gerados quatro trabalhos científicos completos e publicados, sendo:

(i) Managing Virtual Network Embedding through Reconfiguration and Expansion, no journal SIMULATION: Transactions of the Society for Modeling and Simulation International, 2018. Neste artigo o VNE foi abordado em um ambiente SD e online, com a heurística GRASP-RVNS. O foco principal foi a gerência e expansão do $\mathrm{SN}$ a fim de maximizar o número de VNs aceitas. O trabalho propôs mecanismos de reconfiguração, que buscam otimizar o uso do SN e a aplicação de técnicas de detecção de gargalos, que definem pontos de investimentos para a ampliação física do SN.

(ii) Network Embedding in Multi-Domain Environments with Energy Efficiency Concepts, no International Conference on Information Networking, 2018. Neste artigo foi proposta a abordagem heurística GRASPF-RVNS em um ambiente MD e online. O trabalho empregou diferentes objetivos, como maximizar o lucro, minimizar a energia, maximizar o balanceamento de carga e uma versão de otimização híbrida que trabalha com os três objetivos aninhados.

(iii) Abordagens Descentralizadas para o Mapeamento de Redes Virtuais em Ambientes Multidomínio, no Simpósio Brasileiro de Redes de Computadores, 2018. Neste trabalho foi proposta a aplicação da heurística GRASPF-RVNS em um ambiente MD e online, através do controle de um orquestrador. Um estudo comparativo da implementação de três modelos de orquestração distintos foi apresentado. conhecimento total, (ii) parcial e (iii) sem conhecimento da infraestrutura de rede física interna aos domínios. O trabalho explorou métricas como: lucro, consumo de energia e troca de mensagens entre diferentes domínios de rede.

(iv) Modelagens para o Problema de Mapeamento de Redes Virtuais em ambientes Multidomínios, Simpósio Brasileiro de Pesquisa Operacional, 2017. No trabalho foi proposta uma modelagem em PLI, em um cenário MD e online, com diferentes funções de otimização: minimizar a energia, maximizar o lucro, minimizar o overhead de comunicação e maximizar o balanceamento de carga. Ao final do artigo foram comparados os pontos positivos e negativos de cada objetivo.

\section{Trabalhos Relacionados}

Os trabalhos existentes na literatura podem ser classificados em online, periódico ou offline. O modelo offline trata o mapeamento de todas as VNs simultaneamente, tal tratamento pode ser encontrado no trabalho de [Alkmim et al., 2011], que aborda o VNE com Programação Inteira Binária. Demais abordagens offline são vistas em [Leivadeas et al., 2013, Eramo et al., 2016].

No cenário online, não se conhecem as características das VNs que chegam, modelo que melhor carateriza um ambiente de internet, adotado em [Zhu and Ammar, 2006, Chowdhury et al., 2009, Houidi et al., 2011, Beikta et al., 2017]. O trabalho de [Beikta et al., 2017] apresenta uma heurística capaz de atuar nos cenários online e periódico, sendo o modelo periódico processado a cada fatia de tempo decorrido. 
[Houidi et al., 2011] além de trabalhar o modelo online, apresentam uma abordagem periódica baseada em janelas de tempo. Ao final do artigo, [Houidi et al., 2011] concluem que a abordagem periódica consegue melhorar a taxa de aceitação e reduzir o custo de mapeamento em comparação a abordagem online.

O conceito de orquestração de VNs, proposto em [Samuel et al., 2013], atribui a uma entidade, chamada de orquestrador, a função de coordenar o mapeamento de uma VN sobre o SN. Similarmente a [Samuel et al., 2013], neste trabalho foi proposto um orquestrador de redes, mas para ambientes MD e com diferentes objetivos.

Quanto aos objetivos dos algoritmos de mapeamento, diferentes variações são encontradas na literatura: [Houidi et al., 2011] minimizam o custo de mapeamento; [Samuel et al., 2013] maximizam o lucro de cada ISP e minimizam a comunicação inter-domínios; [Zhu and Ammar, 2006] minimizam o estresse dos componentes do SN; [Beikta et al., 2017] maximizam o lucro gerado pelo ISP; e [Alkmim et al., 2011] minimizam a quantidade total de largura de banda alocada no SN para atender as VNs.

\section{Resultados Obtidos}

Nesta seção é apresentada uma análise de desempenho das abordagens avaliadas nos diferentes cenários de teste. Para os testes foram comparadas três diferentes abordagens: ( i) Exata, baseada em PLI, faz uso do solver CPLEX V12.6.3; (ii) Heurística GRASPF-RVNS, baseada nas metaheurísticas GRASP e VNS; e (iii) D-ViNE-LB ${ }^{2}$, abordagem da literatura, baseada em PLI e arredondamento e faz uso do solver GLPK. Ainda foram avaliadas três abordagens de orquestração junto a heurística GRASPF-RVNS. A análise de orquestração variou entre as versões com conhecimento total, parcial e sem conhecimento da infraestrutura de rede física interna aos domínios.

As simulações consideraram quatro tipos de SN distintos: SN1, MD e com uma quantidade exata de recursos exata para um mapeamento ótimo de todas as VNs requisitantes; $S N 2$, MD, com uma sobra de recursos de $25 \%$ para o mapeamento ótimo de todas as VNs; $S N 3$, MD, com uma falta de recursos de $25 \%$ para o mapeamento ótimo de todas as VNs; e por fim o $S N 4$, SD, usado para testes preliminares. Foram considerados três tipos de conjunto de VNs a serem mapeadas: $R 1$, com uma taxa de chegada de VNs de densidade média, usado para analisar a transição entre os modelos online, periódico e offline; $R 2$, com uma taxa de chegada de VNs esparsa, usada para análise do cenário online; e R3, com uma alta taxa de chegada de VNs, usada para análise do cenário online.

\subsection{Análises em cenário SD e Online}

Nos testes realizados de forma online, percebeu-se que a abordagem exata proposta obteve os melhores resultados em termos de aceitação, mas ainda ficou longe do mapeamento ótimo, que só consegue ser atingido com um tratamento offline ou com a aplicação de reconfigurações. Tal fato evidenciou a dificuldade enfrentada pelas abordagens no contexto online. Tal resultado motivou a realização de um estudo comparativo junto aos tratamentos periódico e offline, mostrados na Subseção 5.2.

Outro ponto evidenciado pelos cenários $S N 4-R 2$ e $S N 4-R 3$, foi a baixa aceitação de VNs gerada pela abordagem D-ViNE-LB, se comparada às abordagens propostas

\footnotetext{
${ }^{2}$ Algoritmo determinístico proposto por [Chowdhury et al., 2009], que prioriza o balanceamento de carga no VNE e atua somente em um ambiente online.
} 
neste trabalho. Este fato sugere que o modelo proposto por [Chowdhury et al., 2009] não possui um bom desempenho quando o algoritmo é aplicado em um cenário sem excedentes de recursos no substrato. As abordagens exata e heurística com balanceamento de carga conseguiram uma taxa de mapeamento constante e alta, tanto no cenário de alta entrada e saída de VNs (R3) quanto no de baixa $(R 2)$. Como esperado, as abordagens heurísticas tiveram uma aceitação pior que as abordagens exatas, mas em contrapartida conseguiram ser executadas em um tempo menor, sendo satisfatórias para ambientes online que requerem processamento e mapeamento rápidos.

\subsection{Análises Offline, Online e Periódica}

Analisando-se a aceitação total nos cenários $S N 1-R 1, S N 2-R 1$ e $S N 3-R 1$, percebeu-se que quanto maior o tamanho da janela de tempo do modelo periódico, maior foi a aceitação final das VNs. Este fato ocorreu devido ao processamento das VNs em lote ter trazido um ganho de informação quanto aos recursos demandados, otimizando a utilização dos recursos disponíveis de maneira a ter atendido o maior número de VNs possível. Para uma janela de tempo muito grande, o modelo periódico aproximou-se do comportamento da abordagem offline. Contudo, o cliente conta com um tempo de espera para ter sua conexão atendida, o que pode ser prejudicial em termos de processamento e vazão. Por outro lado, para uma janela de tempo pequena, o comportamento foi similar ao modelo de processamento online, o que pode elevar a taxa de rejeição.

Quando foram examinadas as abordagens que maximizam o lucro de maneira periódica, nos cenários $S N 1-R 1, S N 2-R 1$ e $S N 3-R 1$, percebeu-se que tanto a abordagem exata quanto a heurística, apresentaram uma aceitação total mais baixa que as abordagens que maximizam o balanceamento de carga. Tal fato indicou que quando se procura maximizar a taxa de aceitação final, a função de balanceamento de carga é a melhor opção, justamente por este objetivo promover um uso mais equilibrado de recursos. A função objetivo que propôs reduzir o uso de enlaces inter-domínios causou um impacto negativo em relação à taxa de aceitação final. Este aspecto indicou que mesmo pagando o preço de uma reserva de banda para comunicação entre domínios adjacentes, o mapeamento em mais de um domínio foi necessário para se ter uma aceitação alta.

\subsection{Análise do Orquestrador}

Através da análise nos cenários $S N 1-R 2$ e $S N 1-R 3$, foi notado que o overhead de coordenação causado pela comunicação inter-domínio não foi tão impactante em termos de aceitação. Porém, considerando-se a quantidade de mensagens enviadas/recebidas, poderá haver atrasos no tempo de mapeamento até que a coordenação entre os diferentes domínios seja efetivada. Demostrou-se que a taxa de aceitação gerada pela orquestração com conhecimento total do SN apresentou-se superior as outras variações, justificado, por esta variação aplicar a otimização globalmente nos domínios. Nas demais variações, percebeu-se que a abordagem sem conhecimento do SN gerou uma troca maior de mensagens em relação a abordagem com conhecimento parcial do SN. Com o com conhecimento parcial do $\mathrm{SN}$, o orquestrador já possui uma certa informação que implica na consulta sendo realizada em poucos domínios. Observou-se também que uma maior troca de mensagens propiciou uma melhor coordenação e otimização dos componentes do SN. 


\section{Conclusões}

Neste trabalho foram propostas soluções exata e heurística para resolução do VNE no contexto online, periódico e offline. Além disso, foram tratados diferentes objetivos alinhados com os atuais desafios de virtualização, além de abordagens em único e múltiplos domínios. Por se tratar de um problema de natureza online, as VNs que tem seu tempo de vida finalizado, deixam o SN suscetível a uma possível fragmentação. Por conseguinte, mesmo que existam recursos disponíveis, tais recursos podem se tornar inacessíveis (isolados) para um mapeamento futuro. Quando foi analisada a mudança do ambiente de um único domínio para o de múltiplos domínios, percebeu-se a dificuldade encontrada pelas abordagens para o não isolamento de regiões do $\mathrm{SN}$, dado o baixo número de enlaces inter-domínio. Em trabalhos futuros pretende-se estender e avaliar experimentalmente os modelos propostos para o VNE adicionando conceitos de funções de rede virtualizáveis. Pretendemos ainda aprimorar o modelo de energia, e considerar outros fatores como energias limpas e crédito de carbono.

\section{Referências}

Alkmim, G. P., Batista, D. M., and Fonseca, N. L. S. d. (2011). Mapeamento de Redes Virtuais em Substratos de Rede. Anais do XXIX SBRC, 29.

Beikta, C., Gzpek, D., Ula, A., and Lokman, E. (2017). Secure Virtual Network Embedding with Flexible Bandwidth-based Revenue Maximization. Comput. Netw., 121(C):89-99.

Chowdhury, N. M. M. K., Rahman, M. R., and Boutaba, R. (2009). Virtual Network Embedding with Coordinated Node and Link Mapping. In IEEE INFOCOM 2009, pages 783-791.

Eramo, V., Miucci, E., and Ammar, M. (2016). Study of Reconfiguration Cost and Energy Aware VNE Policies in Cycle-Stationary Traffic Scenarios. IEEE Journal on Selected Areas in Communications, 34(5):1281-1297.

Fischer, A., Botero, J. F., Beck, M. T., de Meer, H., and Hesselbach, X. (2013). Virtual Network Embedding: A Survey. IEEE Communications Surveys Tutorials, 15(4):1888-1906.

Houidi, I., Louati, W., Ameur, W. B., and Zeghlache, D. (2011). Virtual network provisioning across multiple substrate networks. Computer Networks, 55(4):1011 1023. Special Issue on Architectures and Protocols for the Future Internet.

Leivadeas, A., Papagianni, C., and Papavassiliou, S. (2013). Efficient Resource Mapping Framework over Networked Clouds via Iterated Local Search-Based Request Partitioning. IEEE Transactions on Parallel and Distributed Systems, 24(6):1077-1086.

Samuel, F., Chowdhury, M., and Boutaba, R. (2013). PolyViNE: policy-based virtual network embedding across multiple domains. Journal of Internet Services and Applications, 4(1):6.

Zhu, Y. and Ammar, M. (2006). Algorithms for Assigning Substrate Network Resources to Virtual Network Components. In Proceedings IEEE INFOCOM 2006. 25TH IEEE International Conference on Computer Communications, pages 1-12. 